BMJ Nutrition,

Prevention \& Health

\title{
Preventable causes of cancer in Texas by race/ethnicity: insufficient physical activity
}

\author{
Franciska J Gudenkauf (D) , Aaron P Thrift
}

To cite: Gudenkauf FJ, Thrift AP. Preventable causes of cancer in Texas by race/ethnicity: insufficient physical activity. BMJ Nutrition, Prevention \& Health 2020;3:e000087. doi:10.1136/ bmjnph-2020-000087

Department of Medicine, Baylor College of Medicine, Houston, Texas, USA

\section{Correspondence to} Dr Aaron P Thrift, Medicine, Baylor College of Medicine Department of Medicine, Houston, TX 77030, USA; aaron.thrift@bcm.edu

Received 14 April 2020 Revised 14 May 2020 Accepted 22 May 2020 Published Online First 28 July 2020
Check for updates

(C) Author(s) (or their employer(s)) 2020. Re-use permitted under CC BY-NC. No commercial re-use. See rights and permissions. Published by BMJ.

\section{ABSTRACT}

Background According to the 2018 Third Expert Report from the World Cancer Research Fund/American Institute for Cancer Research, there is strong evidence that physical activity of all types and intensities protects against colon, endometrial and breast cancers. We aimed to estimate the percentage and number of incident cancer cases diagnosed in Texas in 2015 that were attributable to insufficient physical activity, and we examined for differences across racial/ethnic subgroups to reveal important causes of and potential avenues for reductions to cancer health disparities.

Methods We calculated population attributable fractions for cancers attributable to insufficient physical activity using prevalence data from the Texas Behavioral Risk Factor Surveillance System and relative risk estimates associated with insufficient physical activity from prior studies. Cancer incidence data were gathered from the Texas Cancer Registry.

Results Overall, approximately $2.0 \%$ of all new cancers or 2094 excess cancer cases diagnosed in 2015 in Texans aged $\geq 25$ years were attributable to insufficient physical activity, with more cancers in women (3.2\%) than in men $(0.8 \%)$. Of all cancer sites, the highest population attributable fraction for insufficient physical activity was observed for endometrial cancers $(21.7 \%$ compared with $12.7 \%$ for colon cancers, $10.9 \%$ for premenopausal breast cancers and $2.0 \%$ for postmenopausal breast cancers). Hispanics (2.6\%) and non-Hispanic blacks (2.5\%) had higher proportions of cancers attributable to insufficient physical activity than non-Hispanic whites (1.8\%).

Conclusions Public health programmes should stress physical activity as a means of cancer prevention, especially among minority groups, who may have disproportionately higher percentages of cancers attributable to insufficient physical activity.

\section{INTRODUCTION}

In 2002, the International Agency for Research on Cancer (IARC) concluded in their Handbooks of Cancer Prevention: Weight Control and Physical Activity that physical activity prevents colon cancer and breast cancer. ${ }^{1}$ At the time, there was limited evidence of a protective effect against endometrial cancer. ${ }^{1}$ In 2014, after re-evaluating the current evidence on the preventative nature of physical activity, IARC recommended with high priority that physical

\section{What is already known on this subject?}

In Western populations, there is strong evidence to conclude that physical activity of all types and intensities protects against colon, endometrial and breast cancers.

- There are limited data describing differences in disease burden attributable to insufficient physical activity according to race/ethnicity.

\section{What this paper adds}

- Minority groups are affected unequally by cancers attributable to insufficient physical activity.

- We found that Hispanics (2.6\%) and non-Hispanic blacks $(2.5 \%)$ had higher proportions of cancers attributable to insufficient physical activity than nonHispanic whites (1.8\%).

- Therefore, prevention programmes should incorporate targeted health promotion efforts that are perceptive of race/ethnicity.

inactivity and sedentary work be considered in the future for the Monographs Programme, which classifies risk factors as carcinogenic to humans. ${ }^{2}$ Further, in 2018, the World Cancer Research Fund (WCRF)/American Institute for Cancer Research (AICR) declared in their Third Expert Report that there is strong evidence that physical activity protects against colon, breast and endometrial cancer, while 'insufficient levels' lead to chronic disease. ${ }^{3}$ As such, it follows that insufficient physical activity may contribute to the burden of cancer in individuals not reaping the protective benefits of regular physical activity. In the USA, previous studies have demonstrated higher levels of insufficient physical activity among Hispanics and non-Hispanic blacks compared with non-Hispanic whites. ${ }^{4}$ Additionally, incidence rates of colon, breast and endometrial cancer in the USA are higher in non-Hispanic blacks, while non-Hispanic whites have the highest incidence rate of all cancers combined ${ }^{5}$; meanwhile, in Texas, 
Table 1 Relative risks associated with category of physical activity level per $250 \mathrm{MET}$-min/week, decreasing from reference

\begin{tabular}{|c|c|c|c|c|c|}
\hline Cancer site & Population & $\begin{array}{l}\text { 750-999 MET- } \\
\mathrm{min} / \text { week }\end{array}$ & $\begin{array}{l}\text { 500-749 MET- } \\
\mathrm{min} / \text { week }\end{array}$ & $\begin{array}{l}\text { 250-499 MET- } \\
\mathrm{min} / \text { week }\end{array}$ & $\begin{array}{l}\text { 0-249 MET- } \\
\mathrm{min} / \text { week }\end{array}$ \\
\hline \multicolumn{6}{|c|}{ Moderate-intensity or vigorous-intensity physical activity } \\
\hline Endometrium $^{10}$ & Women & 1.120 & 1.254 & 1.405 & 1.574 \\
\hline Breast (postmenopausal) $^{10}$ & Women & 1.010 & 1.020 & 1.030 & 1.041 \\
\hline
\end{tabular}

Value in italics means the reported $\mathrm{Cl}$ includes null value.

For moderate-intensity or vigorous-intensity activity, the reference level was $\geq 1000 \mathrm{MET}$-min/week; for vigorous-intensity activity only, the reference level was $\geq 500 \mathrm{MET}$-min/week.

MET, metabolic equivalents of task.

non-Hispanic blacks have the highest incidence rates of all cancers combined and colorectal cancer, while nonHispanic whites have the highest incidence rate of breast cancer. ${ }^{6}$ These differences may suggest that minority groups may be differentially afflicted by cancers attributable to insufficient physical activity, underscoring the need to investigate the role of race/ethnicity in insufficient physical activity-associated cancer incidence. Further, as cancers caused by insufficient physical activity are preventable, this information may be constructive towards improving cancer prevention recommendations.

In this study, we aimed to estimate the population attributable fractions (PAFs) and number of excess cancer cases diagnosed in Texas in 2015 that were attributable to insufficient physical activity. We analysed the cancer types mentioned above (colon, breast, endometrium) for which there is currently strong evidence for a preventative effect (graded as either 'convincing' or 'probable') of physical activity on cancer risk, according to WCRF/ AICR. ${ }^{3}$ For colon cancer, endometrial cancer and specifically postmenopausal breast cancer, the evidence pertains to physical activity of all types and intensities. ${ }^{3}$ There is also strong evidence for protection against premenopausal breast cancer, but only with regard to vigorous-intensity physical activity. ${ }^{3}$ Thus, complying with these nuances, we analysed cancers attributable to only insufficient vigorousintensity activity for premenopausal breast cancer, while broader activity intensities were analysed for colon, endometrial and postmenopausal breast cancers. While the WCRF/AICR report speaks directly about the protective relationship between physical activity and cancer risk, ${ }^{3}$ we were interested in quantifying the converse-the cancer burden attributable to insufficient physical activity, which we have defined as not meeting nationally based physical activity recommendations; thus, the remainder of this analysis will refer to insufficient physical activity. Furthermore, we based our analysis in Texas because the diverse population is conducive to investigating the role of race/ethnicity in cancers caused by insufficient physical activity. To test our hypothesis that insufficient physical activity-attributable cancers may differ across racial/ ethnic subgroups, we further stratified our analysis by race/ethnicity.

\section{METHODS}

The counts of incident invasive cancer cases diagnosed in Texas in 2015 were collected from the Texas Cancer Registry, overall as well as by age group (25-34, 35-44, $45-54,55-64,65-74,75-84, \geq 85$ years), sex and race/ ethnicity (all races/ethnicities, non-Hispanic whites, nonHispanic blacks, Hispanics, other races/ethnicities). ${ }^{7} \mathrm{We}$ identified cancer cases for the current analysis by using the relevant Surveillance, Epidemiology, and End Results Site Recode International Classification of Diseases-O-3/ WHO 2008 definition codes (C18 for colon, C50 for breast and C54-55 for endometrial cancers) ${ }^{8}$ For breast cancer, premenopausal cases included those diagnosed in women aged $<45$ years, and postmenopausal cases included those diagnosed in women aged $\geq 45$ years, which permitted some natural variation around the median menopausal age of 51 and aligned with our age groups (ie, the age group 45-54 years best captured the median menopausal age). ${ }^{9}$

Estimates of relative risk (RR) for insufficient physical activity were derived from a similar PAF study, which reported RRs per 250 metabolic equivalents of task (MET)-min/week ${ }^{10}$; this USA-wide study sourced RR estimates from dose-response meta-analyses of studies conducted in the USA, North America, Europe or worldwide as available and converted published RR estimates to reflect consistent exposure categories of 250 MET-min/ week. ${ }^{10}$ Aerobic physical activity intensity can be measured by metabolic equivalents of task, or METs, which quantify energy expenditure during exercise relative to rest. ${ }^{11}$ The US Department of Health and Human Services recommends in their Physical Activity Guidelines for Americans that adults perform 150-300 min/week of moderate-intensity physical activity or 75-150 min/week of vigorous-intensity physical activity, which both roughly equate to 500-1000 MET-min/week. ${ }^{11}$ For consistency with the USA-wide PAF study's methodology (given that our study population is 


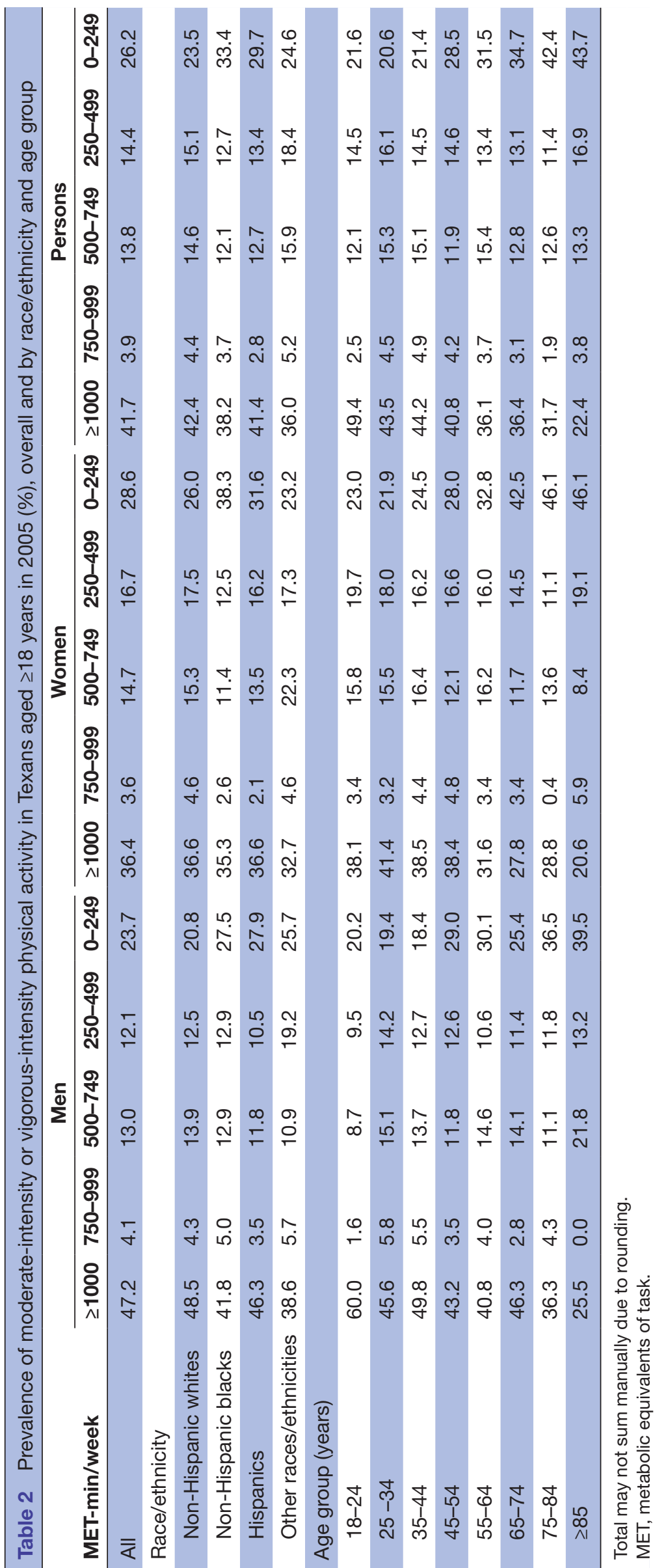


Table 3 Prevalence of vigorous-intensity physical activity in Texan women aged $\geq 18$ years in 2005 (\%), overall and by race/ethnicity and age group

\begin{tabular}{|c|c|c|c|}
\hline & \multicolumn{3}{|c|}{ Women } \\
\hline & $\begin{array}{l}\geq 500 \\
\text { MET- } \\
\text { min/ } \\
\text { week }\end{array}$ & $\begin{array}{l}\text { 250-499 } \\
\text { MET-min/ } \\
\text { week }\end{array}$ & $\begin{array}{l}\text { 0-249 } \\
\text { MET-min/ } \\
\text { week }\end{array}$ \\
\hline All & 28.5 & 6.5 & 65.1 \\
\hline \multicolumn{4}{|l|}{ Race/ethnicity } \\
\hline Non-Hispanic whites & 31.0 & 6.2 & 62.8 \\
\hline Non-Hispanic blacks & 25.8 & 5.4 & 68.8 \\
\hline Hispanics & 24.1 & 6.7 & 69.3 \\
\hline Other races/ethnicities & 28.0 & 13.1 & 58.9 \\
\hline \multicolumn{4}{|l|}{ Age group (years) } \\
\hline $18-24$ & 30.7 & 8.0 & 61.3 \\
\hline $25-34$ & 33.0 & 10.6 & 56.4 \\
\hline $35-44$ & 35.2 & 6.5 & 58.4 \\
\hline $45-54$ & 30.7 & 5.5 & 63.8 \\
\hline $55-64$ & 23.1 & 5.9 & 71.0 \\
\hline $65-74$ & 17.5 & 1.8 & 80.7 \\
\hline $75-84$ & 11.6 & 1.0 & 87.4 \\
\hline$\geq 85$ & 6.1 & 1.2 & 92.7 \\
\hline
\end{tabular}

Total may not sum manually due to rounding. MET, metabolic equivalents of task.

a subset of the US population), we used the upper end of this range as the reference level for combined moderateintensty or vigorous-intensity exercise (1000 MET-min/ week) and the lower end of this range as the reference activity level for vigorous-intensity exercise only (500 MET-min/week). ${ }^{10}$ Physical activity below these reference levels was declared insufficient. Physical activity levels were thus defined by 250 MET-min/week increments, decreasing from reference (moderate-intensity or vigorous-intensity activity: $\geq 1000$ MET-min/week (reference group), 750-999 MET-min/week, 500-749 METmin/week, 250-499 MET-min/week, 0-249 MET-min/ week; vigorous-intensity activity only: $\geq 500$ MET-min/ week (reference group), 250-499 MET-min/week, 0-249 MET-min/week). ${ }^{10}$ Hence, the lowest level of physical activity represents the greatest deficit from reference, or the highest level of insufficient physical activity. Sourced RR estimates were applied to the highest physical activity level below reference (ie, 750-999 MET-min/week for moderate-intensity or vigorous-intensity activity, 250-499 MET-min/week for vigorous-intensity activity); RRs for subsequent activity levels were calculated assuming a constant rate of increase in risk with decreasing physical activity level. Table 1 shows RRs by category of physical activity level.

The Texas Behavioral Risk Factor Surveillance System (BRFSS) provided weighted (to known population proportions via poststratification $)^{12}$ prevalence estimates of physical activity in Texan adults by age group (18-24, 25-34, 35-44, 45-54, 55-64, 65-74, 75-84, $\geq 85$ years), sex and race/ethnicity (all races/ethnicities, non-Hispanic whites, non-Hispanic blacks, Hispanics, other races/ ethnicities) ${ }^{13}$ In accordance with other studies, ${ }^{14}{ }^{15}$ we assumed a 10-year latency period between exposure and outcome. However, BRFSS did not quantify physical activity performed by survey respondents in their 2006 database, so we used data from survey year 2005 . From the BRFSS data, we calculated the average time spent exercising weekly (ie, in min/week) for moderate-intensity activity and vigorous-intensity activity; survey respondents were given examples of non-occupational moderate versus vigorous activities to assist in categorising their intensity. ${ }^{13}$ While WCRF/AICR's conclusions pertained to all activity intensities for colon, endometrial and postmenopausal breast cancer, BRFSS did not quantify low-intensity physical activity, ${ }^{13}$ so moderate-intensity or vigorous-intensity activity was quantified for these cancers. We then converted min/week to MET-min/ week using the previously mentioned conversion and categorised physical activity levels based on MET-min/ week as described above. Table 2 shows the prevalence of moderate-intensity or vigorous-intensity physical activity by activity level, while table 3 shows the prevalence of only vigorous-intensity physical activity by activity level in women.

Standard formulae were used to calculate PAFs by cancer type, age, sex and race/ethnicity: $P A F=\frac{\sum\left(p_{x} * E R R_{x}\right)}{1+\sum\left(p_{x} * E R R_{x}\right)}$, where $p_{x}$ is the population proportion at exposure category $\mathrm{x}$ (category of physical activity as defined above) and $E R R_{x}$ is excess relative risk $\left(\mathrm{RR}_{\mathrm{x}}-1\right) \cdot{ }^{14}$ The resulting PAFs were multiplied by incident cancer counts to calculate the number of excess cancer cases attributable to insufficient physical activity, by sex, age group and race/ethnicity. The latency period was accommodated by pairing prevalence estimates and associated PAFs in each age group with the cancer incidence age group 10 years older (eg, 2005 prevalence data for age group 25-34 years paired with 2015 cancer counts for age group 35-44 years). Because prevalence data were only available for adults 18 years or older, the youngest age group analysed for prevalence was 18-24 years, which corresponded with the youngest age group analysed for incidence of 25-34 years; thus, PAFs were calculated for age $\geq 25$ years. Next, we calculated the age-weighted percentages of incident cancers attributable to insufficient physical activity by cancer type, sex and race/ethnicity. Finally, we added all excess cases across all cancer types to find the percentage of all incident cancer cases (excluding basal cell carcinoma (BCC) and squamous cell carcinoma (SCC) of the skin) diagnosed in Texans aged $\geq 25$ years in 2015 that were attributable to insufficient physical activity.

\section{RESULTS}

In $2005,40.6 \%$ of all Texans aged $\geq 18$ years did not meet the US Department of Health and Human Services' 
Table 4 Observed case counts of colon, endometrial, premenopausal breast and postmenopausal breast cancers, and all cancers combined, diagnosed in Texas in 2015 by race/ethnicity (\%), adults aged $\geq 25$ years

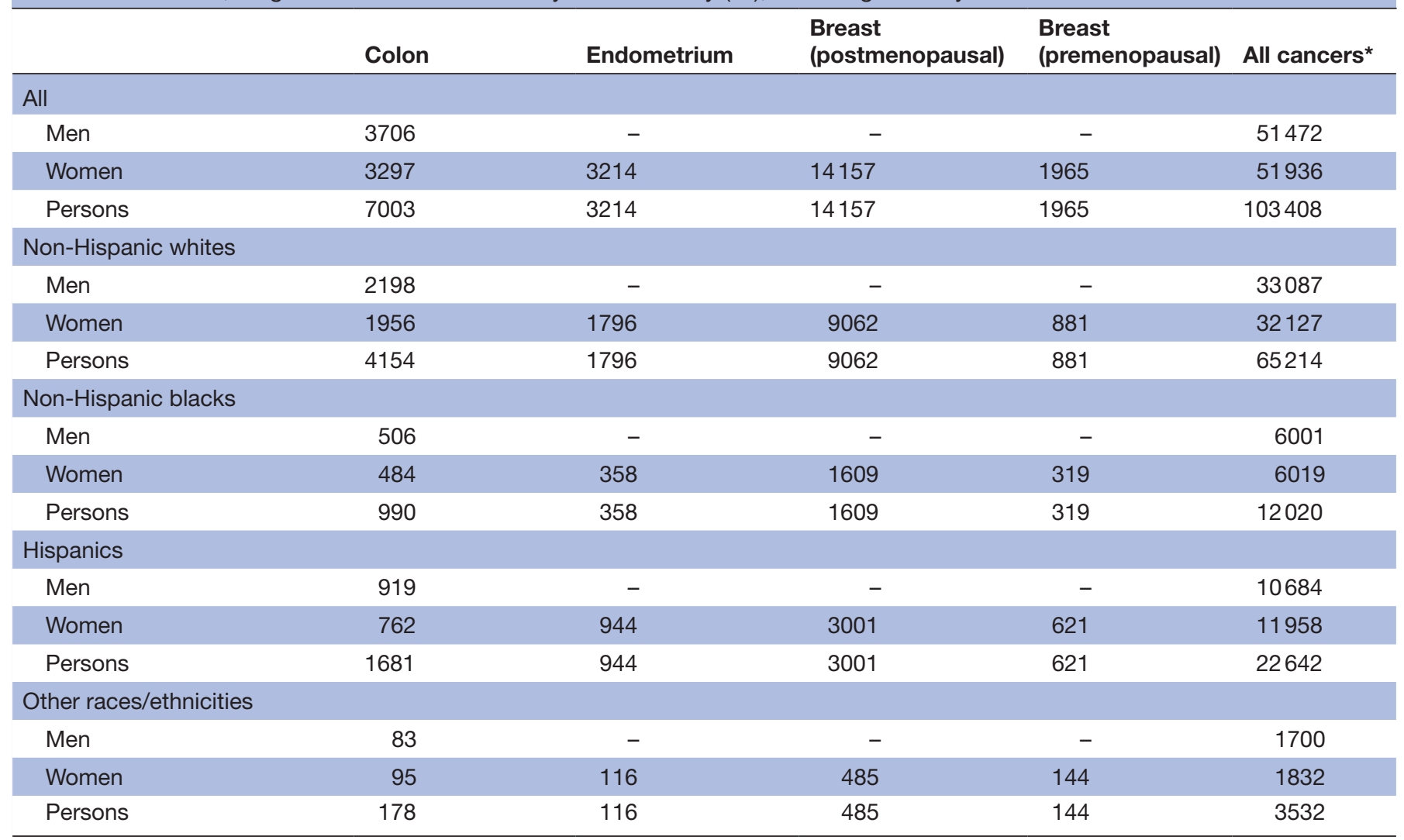

*Excluding basal cell carcinoma and squamous cell carcinoma of the skin.

minimum physical activity recommendation of 500 MET-min/week through moderate-intensity or vigorousintensity exercise (table 2). More women $(45.3 \%$ ) than men $(35.8 \%)$ did not meet these recommendations, a trend that persisted across all age groups. In general, more adults in older age groups did not meet recommendations as compared with younger adults.

More non-Hispanic blacks $(46.1 \%)$ than Hispanics $(43.1 \%)$ and non-Hispanic whites $(38.5 \%)$ did not meet the minimum physical activity recommendation through moderate-intensity or vigorous-intensity activity (table 2). Within each of these major racial/ethnic groups, women were more likely than men to not meet recommendations (women: non-Hispanic blacks, 50.8\%; Hispanics, 47.8\%; non-Hispanic whites, 43.5\%; men: non-Hispanic blacks, 40.3\%; Hispanics, 38.4\%; non-Hispanic whites, 33.3\%).

Through vigorous-intensity activity alone, $71.5 \%$ of Texan adult women did not meet physical activity recommendations (table 3). By race/ethnicity, more Hispanic women $(76.0 \%)$ than non-Hispanic black women $(74.2 \%)$ and non-Hispanic white women $(69.0 \%)$ did not perform adequate physical activity of vigorous-intensity type.

In 2015, there were 103408 cancer cases (excluding BCC and SCC of the skin) diagnosed in Texan adults aged $\geq 25$ years, with nearly equal amounts in men (51472 cases) and women (51936 cases) (table 4). Most cases occurred in non-Hispanic whites (65214 cases), followed by Hispanics (22642 cases), non-Hispanic blacks (12020 cases) and individuals of other races/ethnicities (3532 cases). Overall, 2094 excess cancers or $2.0 \%$ of all new cancer cases (excluding BCC and SCC of the skin) diagnosed in 2015 were attributable to insufficient physical activity (table 5). In women, $3.2 \%$ of all cancers (1659 excess cases) were attributed to insufficient physical activity, while in men only $0.8 \%$ of cancers (435 excess cases) were attributable to insufficient physical activity. Endometrial cancers contributed the highest proportion of excess cancers $(21.7 \%)$, as compared with colon cancer $(12.7 \%)$, premenopausal breast cancer $(10.9 \%)$ and postmenopausal breast cancer (2.0\%). For colon cancer, women had a higher overall site-specific PAF than men (women, 13.9\%; men, $11.7 \%$ ).

Analysis by racial/ethnic subgroup revealed higher proportions of insufficient physical activity-attributable cancers in Hispanics $(2.6 \%, 589$ excess cases) and nonHispanic blacks $(2.5 \%, 298$ excess cases) than in nonHispanic whites $(1.8 \%, 1152$ excess cases). Women had higher overall PAFs than men across all racial/ ethnic subgroups, and endometrial cancer remained the top contributor to excess cancers within each race/ ethnicity. Across the three major racial/ethnic groups, women had higher site-specific PAFs for colon cancer than men, consistent with the results for all persons combined. 
Table 5 Age-weighted PAFs of cancers attributable to insufficient physical activity in Texas in 2015 by race/ethnicity (\%), adults aged $\geq 25$ years

\begin{tabular}{|c|c|c|c|c|c|}
\hline & Colon & Endometrium & $\begin{array}{l}\text { Breast } \\
\text { (postmenopausal) }\end{array}$ & $\begin{array}{l}\text { Breast } \\
\text { (premenopausal) }\end{array}$ & All cancers* \\
\hline All & & & & & PAF (excess cases) \\
\hline Women & 13.9 & 21.7 & 2.0 & 10.9 & $3.2(1659)$ \\
\hline Persons & 12.7 & 21.7 & 2.0 & 10.9 & $2.0(2094)$ \\
\hline Women & 13.6 & 21.1 & 2.0 & 9.7 & $2.8(909)$ \\
\hline Persons & 12.2 & 21.1 & 2.0 & 9.7 & $1.8(1152)$ \\
\hline Non-Hispanic blacks & & & & & PAF (excess cases) \\
\hline Men & 13.0 & - & - & - & $1.1(66)$ \\
\hline Men & 13.7 & - & - & - & $1.2(126)$ \\
\hline Women & 14.5 & 22.7 & 2.2 & 11.8 & $3.9(463)$ \\
\hline Persons & 14.1 & 22.7 & 2.2 & 11.8 & $2.6(589)$ \\
\hline Other races/ethnicities & & & & & PAF (excess cases) \\
\hline Men & 12.6 & - & - & - & $0.6(10)$ \\
\hline Women & 12.5 & 20.8 & 1.9 & 12.0 & $3.4(62)$ \\
\hline Persons & 12.5 & 20.8 & 1.9 & 12.0 & $2.1(73)$ \\
\hline
\end{tabular}

Total may not sum manually due to rounding.

*Excluding basal cell carcinoma and squamous cell carcinoma of the skin. All cancers combined are displayed as PAF (excess cases).

PAF, population attributable fraction.

\section{DISCUSSION}

Our study estimated that $2.0 \%$ of all cancers (excluding BCC and SCC of the skin) or 2094 excess cancer cases diagnosed in Texans aged $\geq 25$ years in 2015 were attributable to insufficient physical activity, with more cancers in women $(3.2 \%)$ than in men $(0.8 \%)$ caused by insufficient physical activity. Of all cancer sites, endometrial cancers contributed the highest fraction of preventable cancers in women $(21.7 \%)$. The percentage of insufficient physical activity-attributable cancers was higher in Hispanics $(2.6 \%)$ and non-Hispanic blacks $(2.5 \%)$ than in nonHispanic whites $(1.8 \%)$.

There is a growing body of evidence showing that insufficient physical activity, especially sedentariness and increased sitting time, is associated with increased risk of developing and dying from cancer. ${ }^{316-18}$ Increasing rates of insufficient physical activity through prolonged sitting and less structured occupational and recreational activities are a concern for population-level cancer prevention. Our results are comparable with a previous USA-wide PAF study which found that $2.9 \%$ of cancers (excluding nonmelanoma skin cancers) in 2014 in Americans aged $\geq 30$ years were attributable to physical inactivity. ${ }^{10}$ Although we applied the same RR estimates to our study as those used in the USA-wide study, the slight difference in the overall PAF in Texas as compared with the USA may be due to differences in prevalence sources and years surveyed. Furthermore, important differences in the demographic characteristics of the Texas population compared with the USA overall (eg, 39\% of Texans are Hispanic compared with $18 \%$ nationally ${ }^{19}$ also contribute to the difference in the overall PAF estimate. The prior USA-wide study did not examine PAFs by race/ethnicity. In the USA in 2014, site-specific PAFs were also comparable with our findings, with endometrial cancer (USA, $26.7 \%^{10}$; Texas, $21.7 \%$ ) contributing the highest fraction of excess cases, followed by colon cancer (USA, $16.3 \%$ (as a per cent of all colorectal cancers ${ }^{10}$; Texas, $12.7 \%$ ) and lastly breast cancer (USA, 3.9\% ${ }^{10}$; Texas, $3.1 \%$ (all breast cancers, data not shown)).

Other studies performed in Australia and the UK in 2010 found that overall $1.6 \%$ and $1.0 \%$, respectively, of cancers in those countries were attributable to insufficient physical activity. ${ }^{14} 15$ All of these studies also found that more cancers were attributable to insufficient physical activity in women than in men (USA $2014^{10}$ : women, 4.4\%; men, $1.5 \%$; Australia $2010^{14}$ : women, $2.9 \%$; men, $0.5 \%$; UK $2010^{15}$ : women, $1.7 \%$; men, $0.4 \%$ ). This is as expected, since colon cancer is the only cancer type analysed that applies to men, and female-specific cancers 
contribute a high burden of cancer incidence to the overall PAF for women (in the current study, endometrial and breast cancers comprised over $70 \%$ of the excess case load in women). In accordance with our study findings, others have also shown a higher site-specific PAF for colon cancer specifically in women than in men (USA $2014^{10}$ : women, $16.8 \%$; men, $15.7 \%$ (as a per cent of all colorectal cancers); Australia $2010^{14}$ : women, $6.9 \%$; men, $6.1 \%$; UK $2010^{15}$ : women, $5.5 \%$; men, $5.1 \%$ ). However, it should be noted that different countries have different national guidelines for physical activity, and thus different reference levels were used across studies, making direct comparisons difficult. ${ }^{14}{ }^{15}$ Yet, despite variations in methodology across studies, our results corroborate previous findings regarding insufficient physical activity as a contributor to cancer incidence, although none of the previous studies has examined PAFs by race/ethnicity.

When stratified by major racial/ethnic subgroups of the population, we estimated higher proportions of cancers attributable to insufficient physical activity in Hispanics $(2.6 \%)$ and non-Hispanic blacks $(2.5 \%)$ than in non-Hispanic whites $(1.8 \%)$. The lower overall PAF for non-Hispanic whites may partially reflect the slightly higher prevalence of adults meeting our reference activity level for moderate-intensity or vigorous-intensity physical activity (ie, $\geq 1000$ MET-min/week) in nonHispanic whites $(42.4 \%)$ as compared with other racial/ ethnic groups, although the differences in prevalence are modest, especially compared with Hispanics (41.4\%). Further, while non-Hispanic blacks $(38.2 \%)$ had the lowest prevalence of adults meeting reference moderateintensity or vigorous-intensity physical activity levels, or the highest prevalence of adults with insufficient physical activity levels, the overall PAF for all cancers was slightly lower in non-Hispanic blacks compared with Hispanics, whose prevalence of sufficient physical activity more closely mirrored that of non-Hispanic whites. Thus, there must be factors other than differences in prevalence-and other than insufficient physical activity-contributing to differences in PAFs across racial/ethnic subgroups.

Our study has several limitations. First, prevalence estimates were derived from self-reported physical activity provided by Texas BRFSS, which may be affected by reporting bias. Although survey respondents were given examples of moderate and vigorous activities to help in their classification, this is inherently subjective and may be inconsistent among subjects. ${ }^{13}$ Further, BRFSS specifically quantified non-occupational moderate-intensity or vigorous-intensity physical activity. Thus, our analysis excludes those in the population who engage in lightintensity or occupational physical activity, and whose inclusion may have affected our results. Also, we partially based our methods off of a prior USA-based PAF study, ${ }^{10}$ using the same physical activity reference levels and categories; while the upper limit of the recommended range of physical activity was used for moderate-intensity or vigorous-intensity physical activity, the lower limit of this range was used for only vigorous-intensity exercise. It can be argued that the same limits of the recommended range should have been used in both instances, but we chose the same levels as the USA-wide study for consistency and comparability. Additionally, while we assumed a latency period of at least 10 years in this study, the real latency period between risk factor and cancer diagnosis is unknown and probably differs by cancer site. ${ }^{14} 15$ Also, while the studies referenced for RR estimates may control for confounding, this population-based study does not exclude individuals with possibly confounding characteristics, similar to previous studies. ${ }^{1415}$ Thus, the potential for confounding may persist. Finally, the category for other races/ethnicities is merely a group for individuals who did not identify with the three major racial/ ethnic groups named in this analysis and may not accurately represent individuals of other named racial/ethnic groups, so inference should be made with caution.

In conclusion, we estimated that $2.0 \%$ of all new cancer cases (2094 excess cases) diagnosed in Texas in 2015 were attributable to insufficient physical activity. Further, Hispanics $(2.6 \%)$ and non-Hispanic blacks $(2.5 \%)$ had higher proportions of insufficient physical activityattributable cancers than non-Hispanic whites (1.8\%). Moreover, Hispanic women $(3.9 \%)$ and non-Hispanic black women $(3.9 \%)$ had greater proportions of cancers caused by insufficient physical activity than non-Hispanic white women $(2.8 \%)$. Thus, women, particularly minority women, could especially benefit from regular physical activity in order to decrease their risk of cancer, although all Texans should strive to meet national physical activity recommendations. Primary prevention programmes should emphasise the protective mechanism of physical activity against cancer development while incorporating information gleaned about racial/ethnic differences in insufficient physical activity-attributable cancers in order to target the most high-risk groups. This may help promote cancer health equity in addition to decreasing future cancer incidence.

Acknowledgements We would like to thank Erin Gardner, MPH, from the Texas Cancer Registry, and Kendall Hammonds, MPH, from the Texas Department of State Health Services, for supplying the relevant public-use de-identified data files analysed in this study.

Contributors FJG and APT planned the study, obtained and analysed the data, and drafted the manuscript.

Funding The authors have not declared a specific grant for this research from any funding agency in the public, commercial or not-for-profit sectors.

Competing interests None declared.

Patient consent for publication Not required.

Ethics approval The research activities of this study were deemed as exempt as data were de-identified and aggregated.

Provenance and peer review Not commissioned; externally peer reviewed.

Data availability statement No data are available. All data are publicly available.

Open access This is an open access article distributed in accordance with the Creative Commons Attribution Non Commercial (CC BY-NC 4.0) license, which permits others to distribute, remix, adapt, build upon this work non-commercially, and license their derivative works on different terms, provided the original work is properly cited, appropriate credit is given, any changes made indicated, and the use is non-commercial. See: http://creativecommons.org/licenses/by-nc/4.0/. 


\section{ORCID iDs}

Franciska J Gudenkauf http://orcid.org/0000-0002-7463-8934

Aaron P Thrift http://orcid.org/0000-0002-0084-5308

\section{REFERENCES}

1 International Agency for Research on Cancer. Weight Control and Physical Activity. In: IARC handbooks of cancer prevention. Lyon, France: IARC Press, 2002. http://publications.iarc.fr/Book-AndReport-Series/larc-Handbooks-Of-Cancer-Prevention

2 International Agency for Research on Cancer. Report of the advisory group to recommend priorities for IARC monographs during 20152019, 2018. Available: https://monographs.iarc.fr/wp-content/ uploads/2018/08/14-002.pdf [Accessed 25 Apr 2019].

3 World Cancer Research Fund/American Institute for Cancer Research. Continuous update project expert report 2018. physical activity and the risk of cancer, 2018. Available: dietandcancerreport. org

4 Saffer H, Dave D, Grossman M, et al. Racial, ethnic, and gender differences in physical activity. J Hum Cap 2013;7:378-410.

5 National Cancer Institute: Division of Cancer Control \& Population Sciences. Cancer race/ethnicity statistics, 2020. Available: https:// surveillance.cancer.gov/statistics/types/race_ethnic.html [Accessed 11 May 2020].

6 Texas Department of State Health Services. Cancer health disparities in Texas by race/ethnicity, 2011-2015, 2018. Available: https://www. dshs.state.tx.us/tcr/data/cancer-health-disparities.aspx [Accessed 11 May 2020].

7 Texas Cancer Registry, Texas Department of State Health Services. Cancer data. Available: https://www.dshs.texas.gov/tcr/

8 Site Recode ICD-O-3/WHO. Definition. National cancer Institute: surveillance, epidemiology, and end results program, 2008. Available: https://seer.cancer.gov/siterecode/icdo3_dwhoheme/index.html [Accessed 22 Apr 2019].
9 The American College of Obstetrics and Gynecology. The menopause years, 2015. Available: https://www.acog.org/Patients/ FAQs/The-Menopause-Years?IsMobileSet=false [Accessed 10 May 2020].

10 Islami F, Goding Sauer A, Miller KD, et al. Proportion and number of cancer cases and deaths attributable to potentially modifiable risk factors in the United States. CA Cancer J Clin 2018;68:31-54.

11 U.S. Department of Health and Human Services. Physical activity guidelines for Americans. 2 edn. Washington, DC: U.S. Department of Health and Human Services, 2018.

12 Centers for Disease Control and Prevention. BRFSS frequently asked questions (FAQs), 2018. Available: https://www.cdc.gov/brfss/about/ brfss_faq.htm [Accessed 22 Apr 2019].

13 Center for Health Statistics (CHS). Texas behavioral risk factor surveillance system survey data. Austin, Texas: Texas Department of State Health Services, 2005.

14 Olsen CM, Wilson LF, Nagle CM, et al. Cancers in Australia in 2010 attributable to insufficient physical activity. Aust N Z J Public Health 2015;39:458-63.

15 Parkin DM. 9. cancers attributable to inadequate physical exercise in the UK in 2010. Br J Cancer 2011;105:S38-41.

16 Biswas A, Oh PI, Faulkner GE, et al. Sedentary time and its association with risk for disease incidence, mortality, and hospitalization in adults: a systematic review and meta-analysis. Ann Intern Med 2015;162:123-32.

17 Ekelund U, Steene-Johannessen J, Brown WJ, et al. Does physical activity attenuate, or even eliminate, the detrimental association of sitting time with mortality? A harmonised meta-analysis of data from more than 1 million men and women. Lancet 2016;388:1302-10.

18 Kerr J, Anderson C, Lippman SM. Physical activity, sedentary behaviour, diet, and cancer: an update and emerging new evidence. Lancet Oncol 2017;18:e457-71.

19 U.S. Department of Commerce. Quick facts Texas; United States, 2018. Available: https://www.census.gov/quickfacts/fact/table/tx,US/ PST045218 [Accessed 8 May 2019]. 